\title{
The Intractable and the Undecidable - Computation and Anticipatory Processes
}

\author{
Mihai Nadin \\ Ashbel Smith University Professor, University of Texas at Dallas; \\ Director, Institute for Research in Anticipatory Systems. \\ Emailid:nadin@utdallas.edu; www.nadin.ws
}

\begin{abstract}
Representation is the pre-requisite for any form of computation. To understand the consequences of this premise, we have to reconsider computation itself. As the sciences and the humanities become computational, to understand what is processed and, moreover, the meaning of the outcome, becomes critical. Focus on big data makes this understanding even more urgent. A fast growing variety of data processing methods is making processing more effective. Nevertheless these methods do not shed light on the relation between the nature of the process and its outcome. This study in foundational aspects of computation addresses the intractable and the undecidable from the perspective of anticipation. Representation, as a specific semiotic activity, turns out to be significant for understanding the meaning of computation.
\end{abstract}

Keywords: Anticipation, complexity, information, representation

\section{INTRODUCTION}

As intellectual constructs, concepts are compressed answers to questions posed in the process of acquiring and disseminating knowledge about the world. Ill-defined concepts are more a trap into vacuous discourse: they undermine the gnoseological effort, i.e., our attempt to gain knowledge. This is why Occam (to whom the principle of parsimony is attributed) advised keeping them to the minimum necessary. Aristotle, Maimonides, Duns Scotus, Ptolemy, and, later, Newton argued for the same. Closer to our time, Kolmogorov (1965) and all those focused on minimal algorithms continue the same line of thought.

When concepts are used to describe specific characteristics of a phenomenon, they ought to be delivered with the criteria for applying them. For example, a square a geometric construct implies four equal sides and 90-degree angles where sides meet. There is nothing open to debate. When making reference to a square, we build upon shared knowledge of geometry. Bit is a contraction for "binary digit." (In another context, it is the cutting edge of a tool.) As a qualifier for data, it describes a variable that, with equal probability, can be zero or one. Once a value is established, we gain information regarding the certainty of the binary random variable.

Data and information are different. Only when meaning is associated with data does it become information. In the quantum realm, the two values associated with a variable exist in superposition; that is, they can be zero and one at the same time. (Of course, for a trinary system of representation, we will have to see how the quantum bit can be reformulated without affecting the broader quantum perspective.)

It is a matter of convention that a group of eight bits is called a byte. A byte is operationally important, but it is not a concept. Concepts are not conventions. They are defined as compressions for knowledge such as the knowledge "contained" in the definition of the square, or of the bit, given above. For quite a number of reasons, some meaningful concepts change over time in order to accommodate new insights. Information 
and cell (in biology) are examples. Compare their current definition with those of 30 years ago and you will notice how much our understanding of what information is and what cells are changed.

Other concepts, generalities mostly (such as matter), remain rather stagnant, in defiance of evidence that undermines their significance: Is a black hole matter or energy? Complexity, in everyone's mouth and under everyone's pen, is also such a concept. Like every concept trivialized through indiscriminate use, it has decreasing resolution power. The undecidable and the intractable, of significance to not only to computation, but also to the science embodied in digital processing, are related to complexity. Therefore, their own definition can benefit from a more rigorous understanding of complexity, provided that it can be subject to operational use (Speculation will not do!).

This study submits a criterion for complexity in the context of science acquired and expressed computationally. It introduces, under the name "G-complexity" G stands for Gödel a clearly defined type of complexity corresponding to decidability (actually, the undecidable), and in direct relation to the intractable. Of course, in science, as well as in life, nobody can "legislate" the use of the word, not to say its meaning. Complexity, understood in so many different ways, will continue to be referred to in many instances where other qualifiers, such as "complication," would be more appropriate. As complicated as some computational processes might be, they nevertheless seem to be decidable because the complicated has only degrees, but not an intrinsic condition of irresolution. Therefore, the particular aspect of complexity, i.e., its reference to the undecidable, expressed in G-complexity, should allow for distinctions of practical consequence. An undecidable process is not less relevant for what we do; the intractable like wise.The practice of scientific inquiry and discovery uncoupled from the validation test in human activity is difficult, if at all justifiable. Of course, one has to be careful. Validation can take some time. Even quantum mechanics required quite a bit of time before validation (the process is far from being exhausted). Never mind number theory, eventually associated with down-toearth methods pertinent to digital security.

The line of argument in this study is straightforward: Science entered a new phase in its development. Computation (algorithmic, interactive, or neural network-based) is now, for all practical purposes, the medium of science. Therefore, to succinctly examine the progress from early computers to quantum computation is to gain access to successive understandings of knowledge acquisition, dissemination, and evaluation in computational form. The challenge of creating forms of computation that can simulate nature (the physical and the living) has epistemological significance. The nature of scientific inquiry has changed as a result of the fact that data processing of bits (or qubits) enables the embodiment of information in the world of new entities: "its" (to restate the famous "Getting Its from Bits" formula of John Archibald Wheeler 1990). 3D printing is only the most recent example of turning bits into new entities of all kind, some leading to new forms of human activity.

With decidability as a well-defined criterion for complexity, we provide a context for understanding how experimental evidence the hallmark of science in our days can be accumulated, and what the characteristics of scientific work are at this juncture in the development of science.

\section{WHAT CAN BE SIMULATED?}

In general, everything that can be modeled. If not entirely, at least partially. Simulations embody knowledge. "...if you want to make a simulation of nature, you'd better make it quantum mechanical," wrote Feynman (1982), arguing for quantum computation. We have come so far. Quantum computation is becoming reality in the form of theoretical contributions the breakthrough work of Deutsch (1985a) and Shor (1994) for example and in the making of physical machines, among them the D-Wave (of Burnaby, Canada). To understand the significance of this implementation, it suffices to mention that Lockheed Martin (with the 
most advanced airplanes, and more than that) became the first paying client. Google is in the process of opening the Quantum Artificial Intelligence Lab, hosted at NASA's Ames Research Center, and run in conjunction with the Universities Space Research Association. All this while scientists still debate whether the D-Wave operates within the quantum domain, more precisely, whether entanglement the signature of quantum processes takes place in the process. Imagine a similar question at the birth of computers: Are they really Turing machines? (We shall return to this.)

Again Feynman: "...we always have had a great deal of difficulty in understanding the world view that quantum mechanics represent." Performance might be impressive. Just one example: in August 2012, a team of Harvard University researchers, solving instances of the Miyagawa-Jerningan lattice protein-folding model on a D-Wave One machine, felt entitled to report on the "largest protein folding problem solved" (Aspuru-Guzik et al. 2012). Still, a question persists: Assuming that entanglement does take place, does it actually lead to the higher performance measured? Aram Harrow's reflections (cf. Aron 2013) on the necessary and sufficient conditions for a true quantum computer cannot be discarded. Others (especially Aaronson 2013) are dedicated to the same subject because it is a matter of scientific relevance. Yes, we should know what is happening in our machines if we really want to understand the meaning of their output.

\subsection{Medium and type of computation}

The question of whether a Turing machine-based computer can, in the final analysis, simulate quantum computation addresses a fundamental problem: Is there a relation between the characteristics of the medium in which computation takes place and knowledge acquired, and the outcome? Or is the Turing machine, with its infinite tape, universal to the extent that it does not matter in which medium it is embodied? We shall argue thatthe nature of computation (algorithmic, interactive, neural network-based, etc.), not the medium of computation (molecule, DNA, silicon, etc.), affects the meaning of output. Simply put: Addition performed with pebbles, on paper, with a calculator or with a computer is not affected by the material but rather by the nature of the operation. It involves representation of what we want to add (numbers as symbols, sensor signals, values in a grid, etc.) and the logic based upon which the operation is carried out. The result is independent of the representation (decimal system, binary, triadic, etc.), but not independent of the notion of addition that governs the operation. The nature of computation (moving pebbles around, writing, etc.) defines its degree of complexity. The Turing algorithmic machine operates in the realm of the complicated; interactive computation, involving the living, corresponds to the realm of complexity.

As impressive as supercomputers are, or as fascinating as quantum computation can be, can it "report" on a domain of reality different in nature from the "physics" it operates on, or is facilitated by? In common jargon: Where complexity often means many components engaged in many interactions, can a low-complexity medium of computation render an outcome pertinent to a higher level of complexity? Wolfram (2002) with his "new kind of science" argues that this is exactly the case (and makes it a "principle of science").

Caveat: The manner of using the word "complexity" in the sense mentioned above is quite contrary to our understanding of the concept. For all practical purposes, we operate in a language domain where this use (right or wrong) is common.

So far, we have made the distinction between complicated (as pertaining to algorithmic computation) and complexity (as definitory of interactive computation). The core of this study, conducted between 1968 and the time of this writing (October 2013), is exactly the question whether computer-based data processing affords an increase from the complicated characteristic of the level of organization at which it takes place (corresponding to the material embodiment of the process) to complexity (that of the biosphere, for example). In an even more drastic formulation: Can complication (corresponding, for example, to the make-up of

Vol. 4, Issue 3, September-December, 2013 (IJARITAC) 
silicon-based machines), as a material substratum for algorithmic computation, facilitate processes that reflect the complexity of the living? In the opening statement of the symposium on "What is Computation?" Denning (2010) took note of "natural information processes," while Wolfram, in the footsteps of Konrad Zuse (1969), had gone so far as to claim that all of nature can be described by simple programs, more precisely by cellular automata. All this certainly reflects yet another thought of Feynman's: "What I cannot create, I do not understand." (This telling statement was apparently on his blackboard at the time of his death in 1988, and was made known by Stephen Hawking 2001). Indeed, my own involvement with computers goes back to 1968 , when a computer meant a CPU with a 60 -bit internal world, chips clocked at $108 \mathrm{KHz}$, and a chip had a mere 2,300 transistors. It extends to the time of the mainframe servers ("big iron") used in time-sharing mode, PCs or Apple computers (Lisa, in particular), client-server configurations, the Connection Machine, DNA computing, etc. The current intelligent agents, games, robots, mobile computation of all kinds, embedded sensors, and the cloud (closing the circle to the horse-powered engines, but now with realtime access) are part of my daily experience as researcher and educator. More recently, a new experience: when to quantum compute means to turn a knob that places the superconducting integrated circuit to a close to zero degrees Fahrenheit temperature. (Way back, I turned knobs on analog computers, but that is a different subject.)

\subsection{Indeterminacy}

At this time of the computer embodied in smartphones, the domain of classical physics (where almost my entire computing experience took place) gives way toquantum reality, and to forms of computation attempting to model life. Until recently, the quantum was the domain of famous Gedanken experiments. (Heisenberg's imaginary gamma ray Gedanken microscope never caught up with me). The values of the bits representing data to be processed on the proprietary chip meant to function in a quantum mode (i.e., entangled) are all that counts. Turn the knob in the opposite direction and the outcome is an energy map expressed as a familiar sequence of zeroes and ones. What would take days, if not weeks, on even the fastest computers available (the supercomputers to which we have access) is resolved in a short time, although not necessarily in the manner we expect. Probabilities sometimes make the turn of the knob lead to puzzling results. There is indeterminacy in the quantum world. The same indeterminacy defines what has become known as "natural and continuous information processes" (Denning 2010), "Evolutionary Computation" (Eberbach 2005), and "Environmental Computation" (Rawbone undated).

\section{TO COMPUTE IS NOTHING MORE THAN TO MEASURE}

Quite a number of sciences, among them genomics, nanoscience, large-scale modeling of weather or of earthquakes, would not exist were it not for digital computation. Astronomers used human beings as computers for their maps before dedicating digital resources to radioastronomy and virtual observatories. Human computations were expected to be tractable (taking finite time) and decidable (the result was well defined). Science being fundamentally a measurement of whatever its object, computation has facilitated forms of measurement otherwise not possible. In order to understand what all this means to improved measurement, let's describe some computations, keeping our focus on complexity and the goal to simulate reality in order to get access to knowledge about it.

The Turing machine is the "mother" of all computers-a blueprint elaborated in mathematical language. All there is to it is a tape, a reading head, and an alphabet of two letters, conveniently selected as 0 and 1. A finite control mechanism reads input data, executes instructions, and reads the output data.

Formally a Turing machine is a 5-tuple

$$
\mathrm{T}=<\mathrm{Q}, \Sigma, \Gamma, \mathrm{q}_{0}, \delta>
$$


Where $\mathrm{Q}$ is a finite set of states, not containing the symbol $\mathrm{h}$ (corresponding to the halt state).

$\Sigma$ is a finite set of symbols and it is the input alphabet.

$\Gamma$ is a finite set of symbols containing $\Sigma$ (as its subset). It is the set of tape symbols.

$\mathrm{q}_{0}$ is the initial state.

$\delta$ is the transition function (but its value may not be defined for certain points).

A Turing machine is a hypothetical mechanism that can simulate any computer algorithm, no matter how complicated it is! (Emphasis on complicated.)

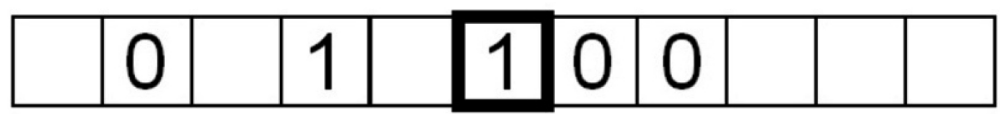

Figure 1: Diagram of a Turing machine

This is a simple diagram of a Turing machine: an infinitely-long tape similar (somehow) to the memory in a computer. The squares on the tape are usually blank at the start. In the machine diagrammed, the machine can only process the symbols 0 and 1 and " "(blank). This would be a 3 -symbol Turing machine.

The Turing machine measures the degree to which logic can prove mathematical assertions. The combination of the theoretic Turing machine (a "mathematical" machine) and the von Neumann architecture (allowing for integrated processing of data and instructions) became the sequential "universal" computer in use today.

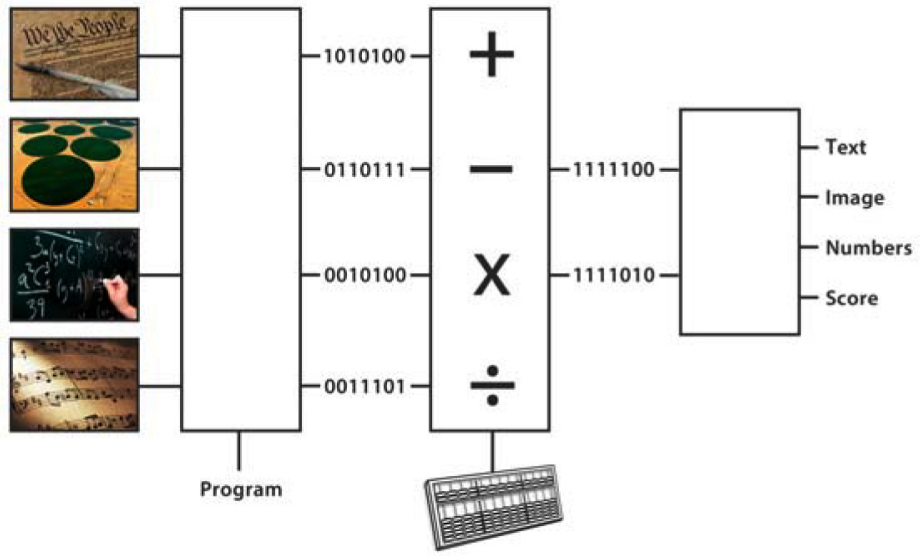

Figure 2: The Turing machine and the von Neumann sequential processing structure embodied in a processing instance. Image, text, numbers, and sounds are encoded in the 2-letter alphabet of the digital machine and subjected to Boolean logic. The abacus symbolizes the arithmetic facility (made available in the hardware). Data is processed regardless of its meaning, i.e., the operation is strictly syntactic.

Leonard Adleman, the "father" of DNA computing, was correct in noticing that Turing's device was conceived for conceptual purposes. One could as well read, instead of zeroes and ones, a string of four DNA letters (A, $\mathrm{T}, \mathrm{C}, \mathrm{G}$ from the genetic code) from the input tape, and write the Watson-Crick complementary string on the output tape. This is what biologists call a "DNA polymerase." The Turing machine proved to be programmable for this language as well, confirming Church's conjecture: A function is machine computable if it is Turing computable. (Gödel referred to "mechanical computability.") 
Adleman (1994) chose the Hamiltonian Path Problem: the sequence of paths to take when one has to travel from place to place (physical addresses for mail delivery, towns for service deliveries, etc.) without going to the same place twice, and, in general, seeking to do it in the shortest time. Computer scientists found many solutions, but none that qualifies as efficient.

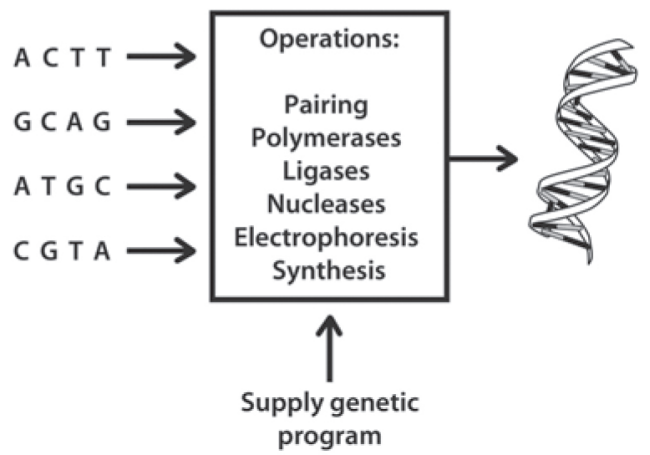

Figure 3: The DNA polymerase machine in action. It isequivalent to a Turing machine. The medium of computation is the test tube and the concentration of DNA (more of an art than science at that time) is the output.

Adleman defined the language the names of the seven cities he chose and associated it with a flight map for the flight connections between cities. The various choices (go cityAcityBcityC, or go cityCcityB, etc.) are expressions in language (sentences). This was called, and still is called, encoding. After that,

"I took a pinch (about $10^{14}$ molecules) of each of the different sequences and put them into a common test tube. To begin the computation, I simply added water-plus ligase, salt and a few other ingredients to approximate the conditions inside a cell. Altogether only about one fiftieth of a teaspoon of solution was used. Within one second, I held the answer to the Hamiltonian Path Problem in my hand," (Adleman 1998).

The Hamiltonian Path Problem is supposed to find an optimal path connecting a point. Underneath lay two deeper questions:

1) What are the best representations of the problem?

2) How long does it take to find the answer?

Adleman ignored the first question, although it was probably more important than the second. When the number of points is large, the possible ways of connecting them becomes exponential. DNA as an alphabet allows for a very efficient "writing" of the output "sentence." Keep in mind: Silicon-based sequential computers driven by algorithm-based programs operating on 2 letters will require a lot more time for this same problem. But, again Adleman: “....although I held the solution in my hand, I also held about 100 trillion molecules that encoded paths that were not Hamiltonian. These had to be eliminated." Yes, the output was a "sentence" in a larger "book" that contained the Hamiltonian path and also all other paths.

The account of this breakthrough computation was read by many, but understood by few. The connection between the DNA model and computer science is the actual subject. Biology, it turns out, is not unlike quantum mechanics in introducing a world view difficult to comprehend. You need to know the language associated with the DNA model and genetic operations. In these days, DNA computing moved from the test tube into living cells. Scientists such as Nadrian Seeman (New York University), Richard J. Lipton (Princeton), and Max G. Lagally (University of Wisconsin-Madison) prepared the way to a DNA-based logic-gate 
operation within a human cell (Hemphill and Dieters 2013). This is not only a technological advance, but also a conceptual breakthrough. Logic gates embodied in strands of DNA have a level of "complicatedness" different from those made up of series of transistors. Adleman's work, and that of the many who follow in his path, are mentioned here only as a preliminary to the question already posed in the introductory lines: Can lower levels of the complicated-ness of the media for computation support processes characteristic of computing that have as outcome knowledge about higher levels of complexity?

In the coming sections, we will focus on the broader interrogation: Can physics-based computation describe the living in a manner that is gnoseologically relevant? Wolpert (2001), addressing the relation between physics and computation, went so far as to ask, "In what sense might reality 'be' a computer?" Many others entertained the same question. (Zuse was convinced that reality is nothing else but the expression of functioning automata). The fact that the living and the physical have a different complexity condition did not necessarily catch the attention of those proponents of reality-as-a-computation (of some kind).

John Preskill, the Richard Feynman Professor of Theoretical Physics at the California Institute of Technology, produced a description for the quantum gate-based computation similar to Adleman's account of his pioneering DNA computing:

"We assemble $\mathrm{N}$ qubits, and prepare them in a standard initial state [...] Then we apply a unitary transformation $\cup$ to the $\mathrm{N}$ qubits [...] After $\cup$ is applied, we measure all of the qubits [...] The measurement outcome is the output of the computation," (Preskill 2011).

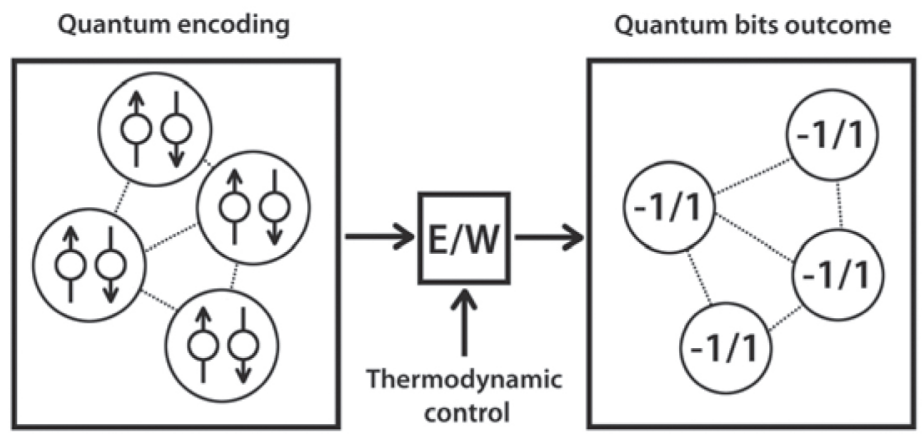

Figure 4: A thermodynamic control process connects the input (presented as quantum encoding) and the output (the quantum bits to be interpreted). This is one possible quantum machine design. Quantum encoding and energy maps are new media of computation.

Just for the sake of a parallel to Adleman's report on his idea, the following from Preskill: "Notice that the algorithm performed by the quantum computer is a probabilistic algorithm. That is, we could run exactly the same program twice and obtain different results, because of the randomness of the quantum measurement." Indeed, the outcome of the DNA computation has its own type of ambiguity. Peterson Ivars (1996) noticed: "It is ... possible that the success of Adleman's beautifully executed experiment can be attributed in part to fortuitous selection of appropriate reaction rates and DNA concentrations."

There is a new quality to the outcome of quantum, or DNA, or membrane, computation: The indeterminacy inherent in the processes they describe is carried over in the outcome. As we shall see, complexity defined in the tradition of simplistically accounting for scale (components and interrelations among them) does not account for this new quality. Mechanical (or machine-based) deterministic operations guarantee the same output for the same data processed. Probabilistic and, furthermore, possibilistic phenomena are less determinate. 


\subsection{Falling in Place}

Against the background of deeply seated scientific methods of computation that are deterministic in nature, imagine someone loading a truck. It takes no time to get the volume of the coal (in whatever units are preferred).
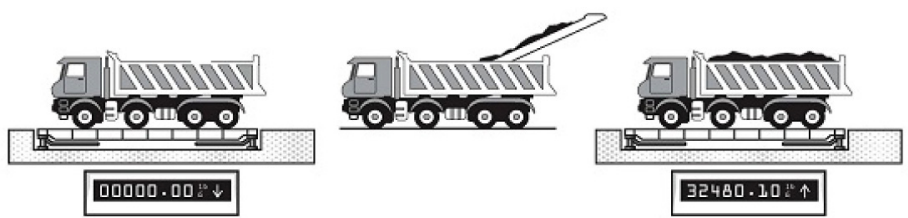

Figure 5: Computing in real time the volume of coal extracted. The weight can also be easily established.

To establish the weight of this volume of coal, one would need the density, and in no time (multiplication is fast) the outcome would be available (again, in whatever units one desires). A scale could do both, combining volume, and weight determination even in the absence of density. These are pretty much like the DNA computation or quantum computing instances of the looked-for value falling in place. Expert abacus users obtain the result in the same way: the beads are moved along and the outcome is the configuration.

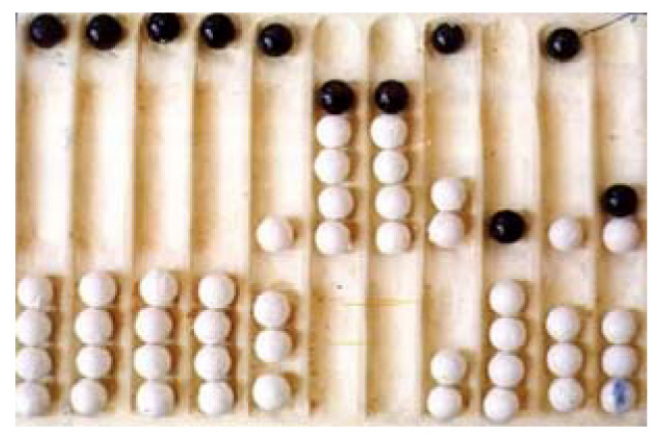

Figure 6: All the arithmetic expected in a computer is available on the abacus.

Try factorization of this outcome, i.e., reverse calculation of the prime numbers multiplied, and you run into a classical tractability problem. If the outcome is of low scale (small numbers), no problem; for larger numbers, Shor (1994) demonstrated that, in principle, a quantum computer, which he never really defined, could do the job efficiently. Factoring is a convenient example of intractable problems. If someone comes up with an answer, obtained efficiently, the results are easy to verify (even on an abacus); the answer to the factorization problem is difficult to reach: you cannot simply guess it.

All this might be of mere documentary interest. The examples given illustrate the thesis that to compute is to measure; moreover, we have illustrations that the outcome of measurement can be simple, complicated, or complex to the extent of being indeterminate. The real epistemological gain is, however, in associating these facts with Feynman's statement: To understand nature, at least at the level of simulating it, one needs to understand quantum mechanics. Previous scientific explanations, embodied in Ptolemy's model of the universe, in Galileo's physics, in Descartes' reductionism and determinism, in Newton's gravity theory, in Einstein's relativity theory (to name those better known), led to claims similar to those that Feynman articulated. Actually, the originators of the quantum mechanics theory were even more given to the scientific conviction that their model was the most encompassing answer to all known scientific questions. If we look at the successive scientific theories, we can, as Kuhn (1962) did, identify paradigms or, on a more intuitive 


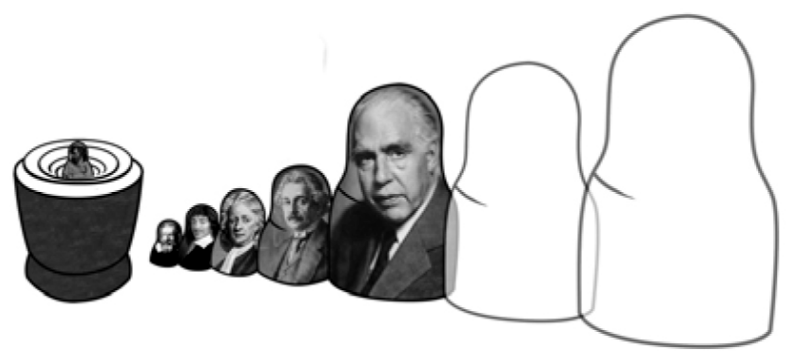

Figure 7: Successive fundamental contributions to knowledge. Given our focus on understanding life, the unnamed next moments might as well be associated with Robert Rosen, or Walther M. Elsasser. But this is not the place for an induction in the "hall of fame" of science.

level, the "Matryoshka" dolls, each fitting into the larger, as though integrating past knowledge into new knowledge.

Feynman taught physics deductively at Caltech, from the level of abstraction of quantum theory to the particular cases of the physics of motion (one Matryoshka doll after another, but in reverse order). To know physics is to practice it. By analogy, to know the language, i.e., to perform in it, is the best way to access knowledge about it. Nobody gets to know a language in a bottom-up procedure, just as nobody acquires competence in physics inductively, one fact after the other. There is no perspective of language at the level of grunts or guttural sounds, or of the alphabet (which is already an abstraction). And there is no perspective of physics (or any science) at the level of apples falling from a tree. Biology cannot be understood by ONLY watching a flower grow. Top-down, deductive thinking, often associated with abduction, i.e., testing hypotheses, gives the broad view, from the height of which the rich domain of how the world is represented in language becomes accessible. Descriptions of reality mathematical, chemical, physical, biological, or even artistic carry over the intrinsic characteristics they stand for. It is in this spirit that Deutsch considers Quantum Theory as a Universal Physical Theory (1985b) and, furthermore, the multiverse as the outcome of quantum computation a claim that goes well farther than Feynman's (And it is in this spirit, as we shall see, that Gödel's undecidable nature of some descriptions comes up).

However, things are never as simple as they seem when broad assertions, such as Deutsch's (and his followers) are made. The Oxford Questions (Briggs et al. 2013) quite properly undermine Feynman's and anyone else's optimistic view. Quantum measurement has so far failed to explain the non-quantum world, that is, "the emergence of a classical world...free of superposition and entanglement." No progress has been made in a quantum theory of gravity, not least because the general relativity, grounded in physical principles, is difficult to reconcile with the quantum mechanics view. Of course, the old shadow is unavoidable as progress is made in quantum computation: What is the upper limit to the scale on which quantum models work?

What the Oxford Questions miss, because their authors work within the tradition of physics, is the realization that the physical and the living are different. Even though Niels Bohr (cf. 1999) and Erwin Schrödinger (1944) acknowledged that life deserves a treatment different from that applied to molecules, energy, etc., they never doubted that, ultimately, quantum mechanics would explain living processes. This is also Elsasser's (1992) point of view. Quantum computation operates under the illusion that it will simulate life because, after all, life is part of the physical reality and does not constitute a domain on its own. That this was never proven does not seem to bother anyone. In order to understand how fundamental the distinction is, let us also examine models of computation and measurement inspired not by particles or waves, but by the living itself. 


\section{THINKING MACHINES WERE NOT THINKING}

Each era has its iconic figures. Feynman was such a figure. Which did not prevent him from asking for a summer job at Thinking Machines Corporation, founded in 1983 by Danny Hillis. (Feynman's son Carl worked there.) The episode is telling. The implicit assertion that brain-inspired computation, i.e., parallel computation, could explain thinking, and thus nature (including quantum mechanics), was provocative. Of course, the effort preceded the attempts at quantum computation. It was based on a simple premise, originating from cognitive science, more precisely, image processing. Those still familiar with printing know that a raster "measures up" an image and translates the continuity (of lines, colors, shapes, etc.) into a substitute (the image is decomposed into squares or circles) good enough to be perceived by the eye looking at a photographically or typographically reproduced image. Computers do the same, as though they would look into the image through a small "peephole."

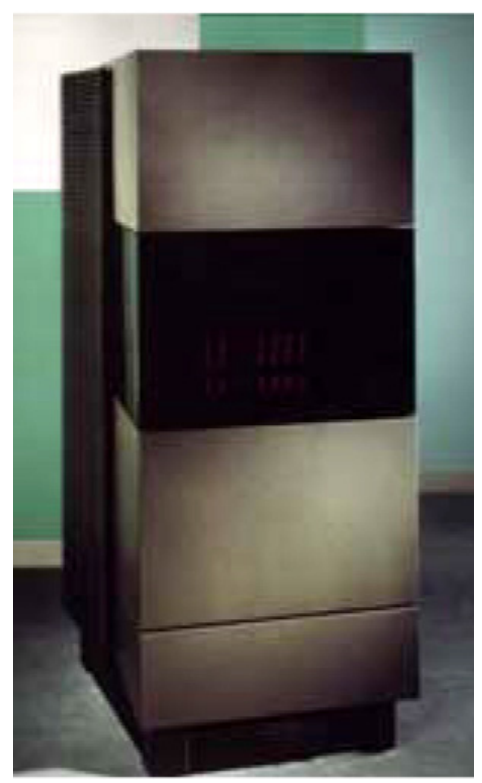

Figure 8: A Connection Machine, designed in order to inspire an intuitive understanding of parallel computation.

A low-resolution reproduction of an image is based on an array corresponding to 256 points on a side. The square consists of 65,536 points, and this was the number of single processors that Hillis used in order to "measure" an image. His argument: On a traditional sequential machine, each of the 65,536 elements of the image is individually evaluated. With his machine, a single processor is assigned to each point. The image is measured at once, as when we measure the volume of coal (as mentioned above), not lump by lump, but together. More important than the parallel architecture (with a very inspired design that made up a 12dimensional hypercube) was the thought that neuronal networks in the brain perform in parallel. Given the cost limitations and the ability to understand the difficulties of programming such a machine, my experience was limited to one quarter of a Connection Machine. The output was a visualization of the famous 2-slit experiment from quantum mechanics.

Together with Thomas Lippert and Norbert Eicker (currently at the John von Neumann Computer Center of the Jüllich Research Center), I debated the relation between anticipatory processes and quantum phenomena while trying to visualize phenomena on a parallel processing machine. 

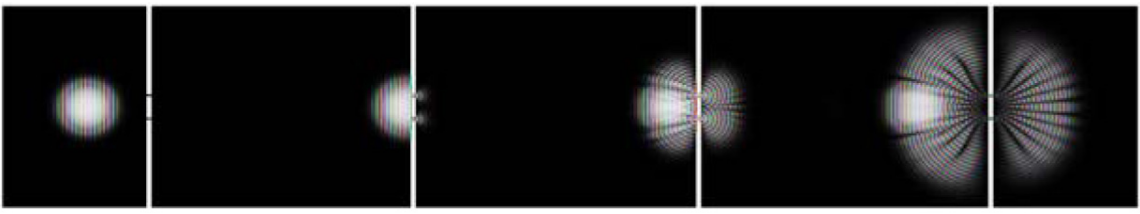

Figure 9: Classic double slit experiment. The image is supposed to provide information about processes that seem teleological (driven by a purpose).

\subsection{Initiation, Generalization, Abstraction}

The main reason for mentioning Hillis (who is still working on a slow-moving device for a clock intended to tick for more than 10,000 years) is not really obvious. His machine did not emulate (i.e., did not imitate) how neurons function. The machine does not pursue the analogy between neuronal activity (in the von Neumann sense) and computation. Rather, he abstracted from neuronal activity a model of segmenting the task (what science sometimes calls fracturing, in reference to reductionism) into a large number of identical operations carried through simultaneously.

Brute force remains brute force, regardless of whether you use a huge cannon or one million smaller guns. Hillis conceived his "cerebral starship" to performon selected projects (mainly image processing) way faster than the supercomputers of his time. Others, inspired by McCulloch and Pitts (1943) and Hebb (1949), felt encouraged to develop neural networks an alternative to algorithmic and sequential computation (the von Neumann machine). This was a breakthrough in terms of advancing models of computation based on understanding how the living processes sensory data. It should be noted that neuronal configurations (the so-called "neuronal networks"), subject to training and learning, circumvent decidability and transcend the intractable.

To train a neural networkis not an expression appropriated mechanicallyfrom human experience. It suggests learning a major human activity extended to the specific realm of interactive computation. At the same time, others (von Neumann among them, 1948, 1951) conceived of artificial neurons. It is in respect to this last detail that I shall address the need to define the notion of complexity in a stricter manner. Just to hint at what I have in mind: A neuron, as part of the brain, and an artificial neuron have quite a number of commonalities. But what distinguishes them, in the final analysis, is that the neuron is part of an irreducible high-complexity network of interactions, while the artificial neuron might be deployed in networks of similar scale, without reaching the same level of complexity. But let's proceed a bit more systematically in justifying the above statement.

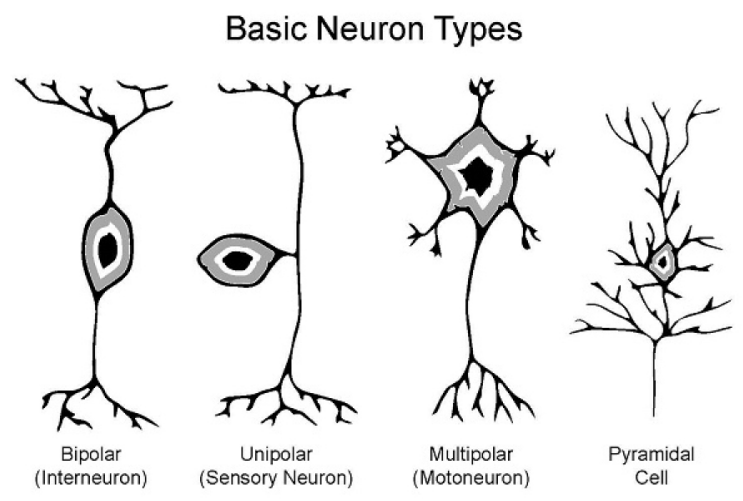

Figure 10: Basic neurons types: an ever-growing diversity. The 3-D representatiion of neurons adds more understanding to how neuronal networks work. 
Quite appropriately, infatuation with neurons, observed in action, informed the attempt to emulate them. Furthermore, this is how artificial neurons became the focus of theoretic and applied science. The approach was driven by the reductionist impetus still dominant in science today: If we know one neuron, we know how any other neuron functions, and how neurons operate together. It was informed by the fundamental scientific ideology: the neuron is a deterministic entity. The two premises were faithfully reproduced in the artificial neuron.

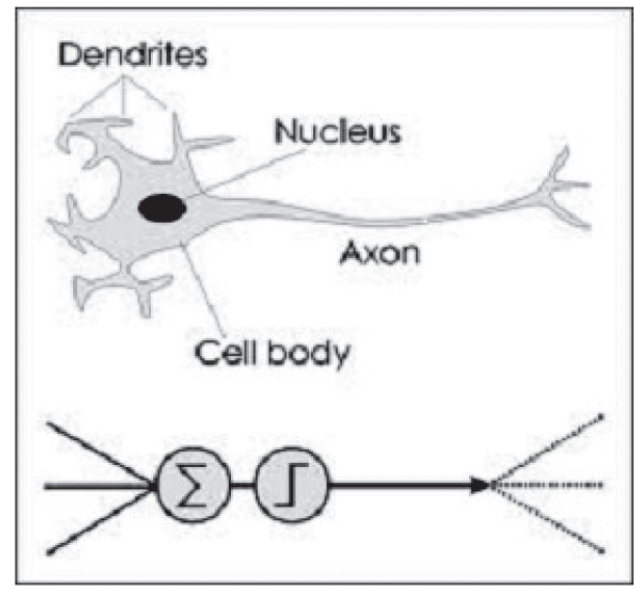

Figure 11: From the neuron to the artificial neuron (cf. Leverington of Texas Tech University and his introduction to feed forward propagation in neural networks).

Of course, the deployment of such a model of artificial neurons in a variety of applications could only confirm the epistemological premises.The "reduction" was good enough for many computations, but inappropriate by far when the task reached levels of complication well above those for which the simplified model can be used. Today we know better; neurons cannot be properly conceived independent of the networks they are part of.

While a major effort to build a "wet computer" that would literally simulate neurons and signal processing on the chemical level is under way, we also learn that neurons are much more than originators of the electrical blips that scientistsrecord. Twitching in dendritic spines, subtle movements of membranes, and the diffusion of water in axons are only three details that debunk the flat model of artificial neurons. What happens when cells fire the metaphor taken over from electricity is also questionable remains mostly unexplained. There is more to the story: "How can a worm remember things after losing its head?" The question was formulated by Tal Shomrat and Michael Levin (2013), who study how animals store and process information. The assumptions of the analogy between machines (computers or simpler devices) and the living are put to question given the amount of evidence to the contrary.

\subsection{Is interaction-free measurement possible?}

Any attempt at measuring something (classic, quantum, biological) disturbs what is measured. It all boils down to the fact that any measurement involves both the measured and the measurer (in whichever form, i.e., observing subject or measuring device working autonomously). Along this line of thought, it is not surprising that, since any computation involves a physical substratum, the outcome is affected by it. The relation between the input and output informs us in regard to the change in the state of the physical device used. This physical substratum can be, as we have seen, a dump truck, a scale, pebbles, an abacus (or its electronic embodiment in a chip), DNA, or RNA, etc. If the chosen device is in the nano-domain (microscopic 
or submicroscopic), the relation between input and output, i.e., the dynamics, is subject to the laws of quantum mechanics. In view of this awareness, it comes as no surprise that a huge effort has been made in engineering new materials adapted to certain computation requirements. For example, logic gates were designed and produced in order to facilitate quantum computation. The first $25 \mathrm{~nm}$ NAND logicgate had 500 Bohr radii (at Intel in 2010), quite appropriate for the quantum scale. In the meanwhile, given the spectacular progress in nanotechnology, more impressive components have been synthesized and tested.

As spectacular as progress in engineering materials is, we cannot avoid the consequences of measurement. "Interaction-free" measurements an idea advanced by Elitzur and Vaidman (referenced by Kwiat and White, 1997-1998) are meant to take advantage of the dual particle-wave descriptions of photons so that the quantum object can be prepared for a superposition of states. The state of the "particle" and that of the light "wave" are entangled. The focus on measurement is indicative of broader questions still unanswered: Even though quantum mechanics is supposed to be an adequate description of nature, why do we not experience quantum superposition in our ordinary existence?

Of course, computer scientists realize that physicists are more and more in the forefront of computer advancement. Physicists are able to understand decidability and tractability well beyond the functioning of a particular device. Indeed, in final analysis, neither psychology nor technology defines the computational process. It is not abstract science on paper. A research team (scientists from China, Canada, and Singapore, Cai et al. 2013) built a simple device that uses entangled photons to solve linear equations. They fired a laser beam at barium-borate crystals in order to generate two pairs of entangled photons. The photons were separated through a beam splitter. These photons were used to represent qubits, processed by an optical circuit. Detected by a sensor, the solution to the equations associated with them came as the state of their polarization. There is, of course, no exact answer (as one expects with a traditional computer), but rather a "most likely" value, actually a series of values. Another team (from Hebrew University in Jerusalem) caused entanglement swapping between photons that never coexisted in time (Megidish et al. 2013). The result might be less relevant to computation than to encryption, but it exemplifies the idea that the characteristics of the medium in which information processes take place affect the process.

This computation, like any other, is performed on a physically manufactured substratum. It is upon this substratum that the logic applied in the process and the representation alphabet (the symbols) are projected. Therefore, the study of how data is processed requires the understanding of the processes taking place in the physical substratum in the specific material. Paul Benioff (in 1982) and Feynman (independently) pointed out that the device embodied in nano-domain entities can serve as the substratum for quantum computation. For whatever it's worth, Poplavskii (1975) and Manin (1980) (both behind the "Iron Curtain" when they advanced their ideas) were earlier in suggesting that fundamental principles be studied in order to exploit the exponential number of states that might become part of a quantum device.

\subsection{It all comes back to mathematics}

Later (1986), Feynman noticed that "...it seems like the laws of physics present no barrier to reducing the size of computers until bits are the size of atoms, and quantum behavior holds dominant sway." The DWave implementation (one of the many tried in our time) is based on processors that perform an adiabatic quantum logarithm. This is based on the adiabatic theorem: A system initially in its ground state tends to stay in this lowest energy state, provided that the Hamiltonian of the system is changed "slowly." Applying this knowledge from thermodynamics helps searching by a quadratic factor over any classical algorithm. It is a quantum local search. For performing it, magnetic coupling of superconducting loops, called vf-squid flux qubits, had to bemade possible. Harmut Neven (Technical Lead Manager, Image Recognition, at Google) gives the following description: "This design realizes what is known as the Ising model...the simplest

Vol. 4, Issue 3, September-December, 2013 (IJARITAC) 
model for an interacting many-body system [...] it can be manufactured using proven fabrication methods." In adiabatic computing, an array of niobium loops, maintained at low temperature in order to obtain superconducting characteristics, eventually settles for the lowest energy point. For this to happen, quantum tunneling is called into operation: the qubits "crawl" through the energy landscape. The fact that they are entangled i.e., linked in quantum mechanics manner (the states depend on each other) is indirectly evinced through qubit tunneling spectroscopy. Functioning at close to absolute zero temperature, the process is sped up by many orders of magnitude.

D-Wave claims that its 512-qubit processor would be 10,000 times faster, for the type of problems (optimization) it was designed to approach, than the traditional processors. This is not a gate-based quantum computer, of the nature of a universal machine (à la Turing) that can be programmed with any algorithm. The D-Wave chips are designed for optimization problems. They work within a specialized computational environment, not a universal computer. To program this machine, you need to know physics, not computer science. Actually, the crisis of physics (at some moment in time, very few students were interested in a career in the "fundamental science") was overcome by its becoming computational.The device spawns new types of computation. To address an optimization problem means to map it into the energy landscape. Geordie Rose, CTO of D-Wave, describes the task on the company's website: "You're making a one-to-one correspondence between a mathematical optimization problem and the actual physical energy of the chip." No computer science program teaches such knowledge. This is amachine conceived and used by mathematicians and physicists.

Among the applications of this new way of measuring reality in order to better understand it: labeling of news stories and images, sentiment analysis (for movies, blog posts, etc.), automatic detection of cars in an image, study of protein folding, etc. The fact that traditional computers (i.e., von Neumann machines) could be used for the same is unquestionable. The fact that the performance of such machines does not match that of the particular adiabatic quantum process is also undisputed. Still, as impressive as all of this is, a question keeps popping up on the memory screen: Is this what Feynman meant when he claimed that only quantum computation can simulate nature? Is this what Deutsch's more radical take (We can quantum compute everything) leads to? If this were the case we could hope that one day we will know everything about the world and about ourselves. The fact that this is epistemologically "non-sense" results from the simple observation that, change being infinite, there is no such thing as a final explanation of anything (the world or some aspect of it) since the explanation itself becomes part of the explained.

For the record: In 2005, Nature (437:6, October) published an entire issue dedicated to computation predictions for 2020. Realistically speaking, the reading of crystal balls (even under the auspices of Nature) is not a gratifying business. But when from the entire collection (under the signature of reputable authors), only one prediction, at most, sounds more convincing, we realize that it is not competence that fails, but the perspective. "Computers for specific applications are likely to come before general-purpose devices. But that doesn't rule out the possibility that we'll all be playing quantum Grand Theft Auto in the near future," according to MIT's Seth Lloyd. He also believes that the universe itself is a functioning quantum device, which has as output ourselves and the world we try to understand. At MIT mechanical engineers dream big.

\section{IT'S ALL ABOUT COMPLEXITY}

Neither the undecidable nor the tractable come up explicitly as new forms and methods for computation are advanced. Somehow, they are implicit in the mathematical descriptions and definitions of complexity. Therefore, it is at this juncture that we'd better return to the notion of complexity that was introduced in the preliminary considerations guiding this study. Evidently, it does not help that the meaning of the word (its semantics) is at best ill-defined. Revisiting dictionaries, old or new, or the etymology, does not really bring 
us further. Leibniz (credited by Weyl 1932, and mentioned in more than one of Chaitin's lectures, cf. 2006) seems among the first to examine our descriptions of reality from a complexity perspective. Laws ought not to be arbitrarily complex, because if they are, the concept of the law becomes inoperative. In more recent times, Henri Poincaré (1905), and, closer to our time, Ilyia Prigogine (1997) expressed interest in prediction (relation to future) as it is related to complexity (they are mentioned in connection to "chaos" theory, i.e., dynamic systems theory). Economists, such as the political economy of the Scottish Enlightenment, the Austrian School of Economics, as well as Friedrich Hayek (also looking into psychology, biology, and cybernetics), Gregory Bateson, (looking into culture), and, closer to our time, von Neumann, John Holland (1995), and Waldrop (1993) contributed to the interest in the subject of complexity. The Santa Fe Institute, the "Mecca" of complexity studies, inspired similar institutional focus on the subject (cf. Gell-Mann 1995). The indiscriminate use of the concept spawned a large production of pseudo-complexity subjects, including the misnomer "Computational Complexity," standing for efficiency rather than for characteristics of the process. From my perspective, Robert Rosen (1988) remains the closest reference to the complexity intrinsic in living phenomena, not to their descriptions. In his view, there is no largest model for complexity; a complex entity (one would say "system") is not fragmentable (it cannot be subject to reduction). In other words, there are no levels of complexity: you are prequant or not!

On the pragmatic level, complexity is associated with efficiency, in particular, with efficient computing. Nobody ever stated, for example, that the intractable (i.e., a computing task that would require resources and time beyond what is currently available) might somehow define, in negative terms, a goal outside the attainable. This goal would be something so complex that it cannot be performed at current levels of theoretic knowledge and technical know-how. In fact, the intractable is an expression of the limits of computation (sometimes understood in a mechanistic view as memory- and speed-related). The NSF, usually trailing way behind new ideas and concepts, is funding a center (at Princeton University) focused on the subject. Moreover, the Clay Mathematics Institute (Cambridge, UK) is offering a one-million dollar prize (the Millennium Award) to whomever can prove that problems apparently unsolvable with current or scaled-up computational means are intractable. In a different study (Nadin 2014), I hope to lay claim to this prize connecting complexity to tractable computation.

On the other hand, there is the quasi-unanimous recognition of accomplishments associated with complexity. Creativity is a good placeholder and qualifier for the exceptional as an expression of complex processes, by no means associated with a large scale: not big data, rather, scarce, significant data. Actually, creative acts involve little data, just as survival is a small but significant data endeavor. Just for the sake of the argument, one simple example:

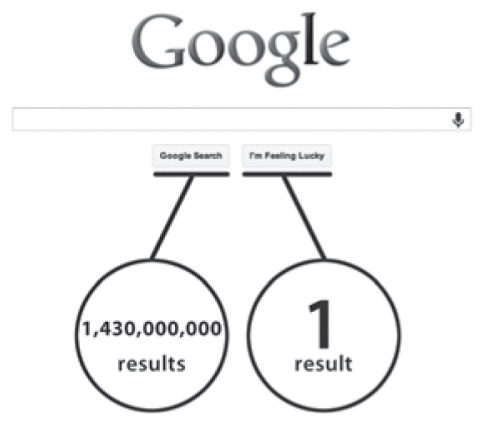

Figure 12: The current obsession with big data corresponds to an understanding of the complicated physical world, not the particular complexity of the living. Progress in computation made the big data frenzy possible. This is an example of technology driving the gnoseological instead of reflecting its purpose. Google recently started to provide "predictive" information.

Vol. 4, Issue 3, September-December, 2013 (IJARITAC) 
All the examples given so far document how computation evolved, sometimes in order to address challenging scientific questions, other times to affirm its new role in science and society. Even the reductionistdeterministic model, which ultimately proved useful in conceiving a new world of artifacts of extreme performance, is an expression of the attempt to understand which representations best support knowledge acquisition attempts. The goal of explanations, i.e., measurement performed under the guidance of reductionism and determinism, is to gain access to knowledge about phenomena otherwise difficult (if not impossible) to explain.

With all this in mind, that is, with respectful acknowledgment of what reductionism and determinism made possible the whole universe of all our technology, and a large body of knowledge informing human activity the need to define complexity, and to introduce a clear criterion (or criteria) for it is more urgent than ever before. Quantum Complexity (complexity understood as computation efficiency) so often extolled, really moved the goalpost in ascertaining that quantum processes are way faster and more encompassing than those we know from classical physics. But the focus has remained on scale, i.e., on quantity, while complexity actually defines quality. Moreover, complexity is definitory of the world in which we live and to which we belong, and which we try to better understand. It can be, of course, an attribute of descriptions of existence. Leibniz (1714) thought about "very complicated" formulations and dismissed them. However, complexity, as consubstantial with existence, is of higher order consequence. If reality, or at least part of it, is complex, the issues involved in knowing this reality (for instance, describing it computationally in its complexity) is of extreme practical importance.

What follows in the line of argument is a proposal that, realistically speaking, will not change the way the concept is used. None other than a logician in the guise of a writer for children (or posing as one while actually addressing us all) wrote:

"When I use a word," Humpty Dumpty said, in rather a scornful tone, "it means just what I choose it to mean - neither more nor less."

"The question is," said Alice, "whether you can make words mean so many different things."

"The question is," said Humpty Dumpty, "which is to be master " that's all."

(Carroll 1871)

In programming languages, words mean only one thing: there is no room for ambiguity, and, obviously, none for poetry.

\section{THE UNDECIDABLE AS CRITERION OF COMPLEXITY}

\subsection{Thesis: A G-complex system is Gödel undecidable}

In order to give this thesis a context, let us recall, in the words of Kleene (1967, p. 250) that

Any effectively generated theory capable of expressing elementary arithmetic cannot be both consistent and complete. In particular, for any consistent, effectively generated formal theory that proves certain basic arithmetic truths, there is an arithmetical statement that is true, but not provable in the theory.

Gödel's own formulation (1931) is quite a bit more elaborate (The effort involved in following his logic is by no means trivial). Of course, his subject is not the world, not Nature (to return to Newton's and Feynman's preferred term for reality), but the dyophantine number theory, i.e., arithmetic. Or better yet: mathematics subjected to the question of mechanical resolution (the Entscheidungs problem that both Turing and Gödel examined). 


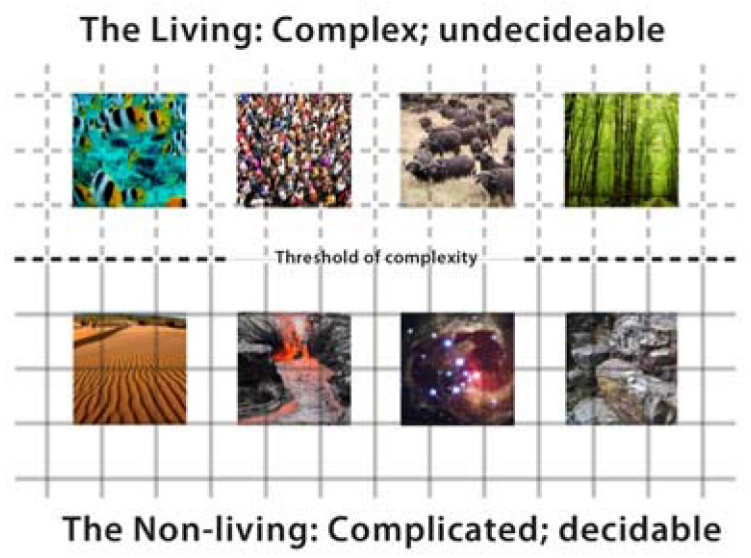

Figure 13: The decidable as criterion for complexity

In more words than the formulation of an effective criterion allows: G-complex phenomena are not fully and consistently describable:

The immediate consequence is that phenomena of G-complexity escape both reductionism and determinism as descriptions of their causality. There is no effective decomposition rule; the consistency clause ascertains that inferences implicit in determinism (same cause $\rightarrow$ same effect) do not hold for the G-complex. They are not cause-free; rather, causality is expressed in forms that no longer submit to the time sequence characteristic of determinism. The future (as we shall see) is constitutive of their dynamics driven by goals (often expressed as intentions). Feynman's Original State Diagram (Fig. 15), to which we shall return, suggests that states in a complex system could depend on both past and future.

Once again: Feynman et al. lay claim to quantum computing as the only way to simulate nature, while they actually ignore a major distinction within this comprehensive Nature: that between the living and the physical. Moreover, they affirm that what holds true for the physical (inanimate reality) holds equally true for life. Among the many who argued in favor of this distinction (all discarded as animists) there are even physicists, not to mention biologists, mathematicians, and, more recently semioticians. The arguments, discussed in detail (Nadin 2003a, b; 2010a, b) support the complexity criterion introduced above.

Of course, the criterion of complexity now becomes, within the inference loop advanced, an identifier of the living. There is no need for animus, or vital substance, or anything of the sort. The living is G-complex. This declaration sounds almost trivial. And this holds true for every living form, whether endowed with one neuron or 120 billion neurons, whether consisting of one reproducible cell or an enormous number of cells of all kinds. The image above (Fig. 13) is, obviously, more an illustrative than a formal argument. Its operational consequences are implicit. Therefore, I suggest the following shorthand for implications of my thesis:

I. Decidability is the criterion for a new type of complexity: the G-complexity.

Definition: A G-complex system is undecidable.

Corollary: Below the complexity threshold is the domain of the complicated and the simple.

Above the threshold, there are no degrees. A system is undecidable or not, i.e., G-complex or not. There is no room for equivocation. 

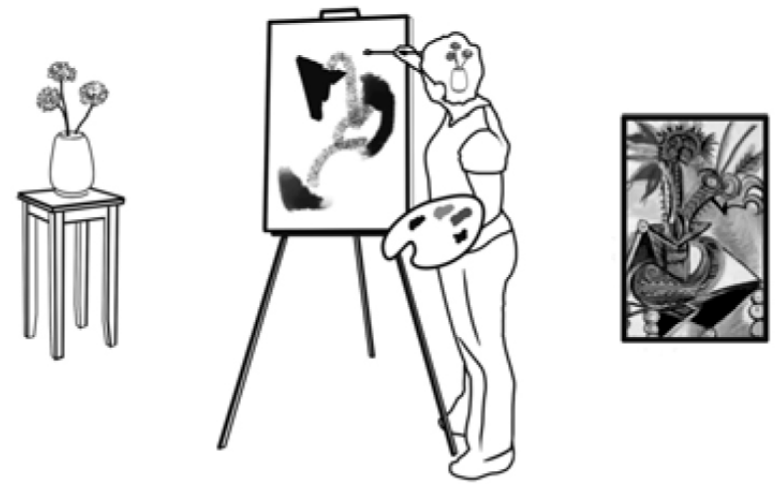

Figure 14: The living generates information.

II. A G-complex system is characterized by the fact that its information level is always above the information received from the environment; that is, it generates information.

Of course, what an artist "takes in" from reality, i.e., the perception, is quite different from what, on account of creativity, is expressed in the work. The illustration could as well have represented a scientist, or a living monocell.

III. G-complex systems are interactive adaptive systems; physical systems are reactive. In adaptive systems, sensory input informs actions, integrating experience (the learning component). Reactions take place on account of received sensorial input. Adaptive processes are not reactive; they involve the future.

IV. A G-complex system is not measurable.

A G-complex system is represented by the life record of the system. Time series can capture partial knowledge about specific aspects of the dynamics qualified through partial measurements. (For example, after surgery, physicians measure temperature, heart rate, blood oxygen levels, etc. These variables represent only a limited aspect of the patient's state of health.)

V. G-complex systems have no effective copy procedures. Everything in a G-complex system is unique.

The knowledge domain of entities and phenomena characterized by G-complexity is the idiographic (Nadin 2012). For any such entity $E_{i}$, always different from any other, we can define a functional dynamic

$\mathrm{y}_{\mathrm{Ei}}=\mathrm{f}_{\mathrm{Ei}}\left\{\mathrm{x}_{\mathrm{Ei}}(\mathrm{t}), \mathrm{I}_{\mathrm{Ei}}(\mathrm{t}), \mathrm{t}\right\}$

Take note that the function is entity specific $\left(\mathrm{f}_{\mathrm{Ei}}\right)$. Interactions $\left(\mathrm{I}_{\mathrm{Ei}}\right)$, part of the dynamics, are also specific. The fact that the function is entity specific excludes generalizations. Evidently, the specific dynamic of one identity actually differs in indeterminate ways from the dynamic of any other entity. The aggregate value is therefore meaningless. G-complex entities do not accept nomothetic descriptions.

VI. G-complex systems are relational.

$E_{i} R_{i j} E_{j}$

Living entities are interrelated. The mathematical descriptions through relations have to acknowledge the variety of possible relations and how they switch from one form to another in the interaction. 
VII. G-complex systems are endowed with self-evolving anticipatory processes.

An anticipatory system is characterized by:

$x(t)=f(x(t-\alpha), x(t), x(t+\beta)$

where $x(t-\alpha)$, defines a previous state, $x(t)$ is the current state and $x(t+\beta)$ is a future state.

The convenience of the standard $(\mathrm{t}-1)$, or $(\mathrm{t}+1)$ for describing past and future must give way to descriptions of a finer granularity ( $\mathrm{t}-)$ respectively $(\mathrm{t}+\alpha)$, where $\alpha$ and $\beta$ might be of different scales.

In other words: The current state of an anticipatory system depends upon previous states, its own condition at the time of determination (measurement), and possible future states. One can say, in the jargon of quantum mechanics, that past, present, and future are entangled. A G-complex system is an evolving record of entangled (not quantum entangled though) past states, current states, and possible future states. For an observer, actions are the expression of anticipation. G-complex systems are open systems, of unlimited dynamics.

The physical world, such as the world on Mars (devoid of life, as far as we know) runs the gamut between the simple and the complicated. Complicated systems are made of simple systems, or can be reduced to a limited number of simple systems.Complicated systems are subject to observation and measurement. To know such a system is to capture its regularity, obvious or hidden. This regularity corresponds to the laws predicting the behavior of such systems. They belong to the knowledge domain of the nomothetic (Nadin, 2010a, b). Complicated systems are closed systems, of limited dynamics. The experimental method as a source of knowledge about the physical is based on the assumption that competing explanatory models (i.e., hypotheses) can be empirically tested. This is the basis for the expectation of repeatability. The domain of the physical (inanimate) allows for this procedure.

Experiments always being closed systems within which variability (of parameters) can be quantitatively described, it is reasonable to rely on them for knowledge acquisition. To predict the behavior of a complicated system, the observer requires sufficient information about its dynamics. There cannot be enough information about a G-complex system, since it produces information as it evolves, adapts to a variable world, and interacts with it. Life is interaction. Therefore, the experimental model pertinent to the domain of the decidable (we can fully describe such entities contradiction-free) is not applicable in the G-complexity domain. The fact that it is practiced corresponds rather to the politics of science than to intrinsic scientific aspects. Even generalizations built upon statistical averages and probability distribution defy the nature of the entity subject to knowledge acquisition. Quite often, the irreducible uniqueness (of human subjects, or of animals) is replaced by a conditioning procedure.If nothing else, the implications for research of the complexity criterion advanced bring into focus the hot issues of the intractable.

\subsection{Thesis: No other medium, except the living, is above the G-complexity threshold}

Corollary: The output of any physical systems-based form of computation (in silicon, quantum, in DNA, RNA, etc.) is by definition under the threshold.

Ergo: The living cannot be simulated by computation in a non-living substratum.

The entire effort of embedding computation in artificial entities emulating aspects of the living (synthetic neurons, artificial muscles, synthetic DNA, synthetic cells, etc.) deserves respect for the gnoseological, scientific, and technological effort. However, the outcomes of the computation on such substrata can only reflect the assumptions embedded in the emulated synthetic world. It is not wrong to generate partial descriptions of G-complex phenomena, as long as they are presented together with the particular metrics adopted. To identify the perspective helps in understanding the meaning of the outcome.

Vol. 4, Issue 3, September-December, 2013 (IJARITAC) 


\section{ANTICIPATION AND G-COMPLEXITY}

Systems that can make predictive models of themselves and their environment, and use those models in directing their actions, are anticipatory (Rosen 1985; Nadin 1986/1991). An anticipatory system is one whose current state depends not only on previous states, but also on possible future states. Anticipation is a corollary of G-complexity.

While Rosen (1985) and Nadin (1986-1991, 2003) are credited for introducing the subject of anticipation to modern science both defining anticipation in contradistinction to reaction it is worth adding to their view that of Feynman (1982) and his Original State Diagram:

$$
s_{i}=F_{i}\left(s_{j}, s_{k}, \ldots\right)
$$

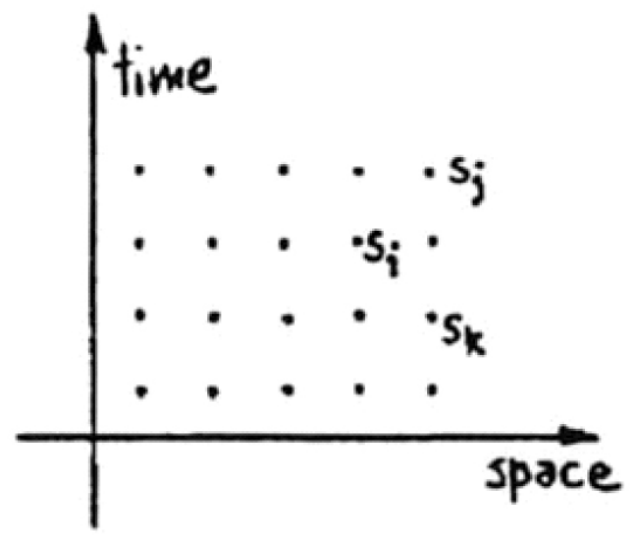

Figure 15: In an anticipatory system, the current state of the system depends not only upon previous states but also upon possible future states (Nadin 2003a).

He suggests: "Just let's think about a more general kind of computer . . . . If F depends on all the points both in the future and the past, what then?" Had Feynman posed this rhetorical question within the context of our research, the answer would be: If indeed $\mathrm{F}$ depends on all the points both in the future and the past, then $\rightarrow$ Anticipation. Indeed, if we work with the definition "a system whose current state depends not only on a previous state and the current state, but also on possible future states," we are within the description given by Feynman: "That could be the way Nature works."

Nature embodies the undecidable in processes characteristic of the living. Our attempts to reduce their undecidable nature and to reconstruct nature as computation (algorithmic, in particular) has generated a large body of contradictory knowledge (in the form of models and simulations) about closed systems of what in reality are open systems. Nature is also intractable, in the sense that to know the living is to live each particular instantiation of life.

\section{ACKNOWLEDGMENTS}

For a study extending over a long timespan, to acknowledge everyone with whom I interacted in the process is a difficult task. I shall restrict the naming to those who challenged my views instead of politely accepting them: Marcian Gutman, Ioan Agarbiceanu, Edmond Nicolau, Grigore Moisil, Wolfgang Stegmüller, Umberto Eco, Solomon Marcus, Max Bense, Heinz von Foerster, Andy van Dam, Douglas Englebart, Terry Winograd, Robert Rosen, Terrence Sejnowski (recently elected to the AAAS), Aloisius Louie, Otthein Herzog, Lotfi 
Zadeh, George Klir, Jonas Salk, Herbert Simon, Hiroshi Kawano, Klaus Schilling, Thomas Lippert, Benoit Mandelbrot, and Stephen Wolfram. My lecture (What is and what is not anticipation, 2013) at UT-Dallas gave rise to many questions from which, I hope, this text benefited. Cassandra Emswiler assisted me in many ways (her illustrations are always in dialog with my thoughts); and so did Elvira Nadin, by now an expert herself in anticipatory systems. John Raven (fighting his many curses) added to the visual argument. Last but not least, the Study Group at the Hanse Institute for Advanced Study/Hanse Wissenschaftskolleg gives me the opportunity to work with many young and very young researchers who, most of the time, know so much more than I do.

\section{REFERENCES}

Aaronson, S. Quantum Computing Since Democritus. Cambridge: Cambridge University Press, 2013.

Adleman, L. Molecular Computation of Solutions to Combinatorial Problems, Science (November 1994), Vol. 266, pp 1021-1024, 1994.

Adleman, L. Computing with DNA, Scientific American, Vol. August 1998, pp 54-61, 1998.

Aron, J. Controversial quantum computer aces entanglement tests, New Scientist (13 March 2013) Vol. 2908, p 3, 2013.

Aspuru-Guzik, A., A. Perdomo-Ortiz, N. Dickson, M. Drew-Brook, G. Rose. Finding low-energy conformations of lattice protein models by quantum annealing, Nature, Scientific Reports 2:571, August, 2012.

Benioff, P.A. Quantum mechanical Hamiltonian models of discrete processes that erase their own histories: Application to Turing machines, International Journal of Theoretical Physics, Vol. 21: No.3-4, pp 177-201, 1982.

Bohr, N. Complementarity Beyond Physics (1928-1962), Niels Bohr - Collected Works, 10. Amsterdam: North Holland, 1999 (See also: Atoms and Human Knowledge, a lecture delivered at the University of Oklahoma-Norman, in 1957).

Briggs, G.A.D., J.N. Butterfield, A. Zeilinger. The Oxford Questions on the foundations of quantum physics, Proceedings of the Royal Society A, Vol. 469, 2013. Published 3 July 2013 doi: 10.1098/rspa.2013.0299 Proc. R. Soc.A8 September 2013, 469:2157, 20130299. Retrieved on July 182013 from http:// rspa.royalsocietypublishing.org/content/469/2157/20130299.abstract

Cai, X.D. et al. Experimental Quantum Computing to Solve Systems of Linear Equations, Physical Review Letters, Vol. 110, p 23, 2013. Retrieved July 18, 2013, from http://prl.aps.org/abstract/PRL/v110/i23/e230501

Carroll, L. Through the Looking-Glass, and What Alice Found There. London: Macmillan, 1871.

Chaitin, G. Epistemology as Information Theory, Collapse, Vol.1, no.8, pp 27-51, 2006.

Denning, P.J. What is Computation, Ubiquity, November 2010. New York: ACM Publishing, 2010. Retrieved on October 25, 2013 from: http://ubiquity.acm.org/article.cfm?id=1880067.

Deutsch, D. Quantum Theory, the Church-Turing Principle and the universal quantum computer, Proceedings of the Royal Society of London, Series A, Mathematical and Physical Sciences, Vol. 400, no.1818, pp 97-117, 1985a.

Deutsch, D. Quantum theory as a universal physical theory, International Journal of Theoretical Physics, Vol. 24, no. 1, pp 1-41, 1985b.

Eberbach, E. Toward a theory of evolutionary computation, Biosystems, Vol. 82, pp 1-19, 2005.

Elsasser, W. Reflections on the Theory of Organisms. Holism in Biology. Baltimore: Johns Hopkins University Press, 1998 (originally published in 1987 by ORBIS Publishing, Frelighsburg, Quebec)

Vol. 4, Issue 3, September-December, 2013 (IJARITAC) 
Feynman, R. Simulating Physics with Computers, International Journal of Theoretical Physics, Vol. 21, no. (6/7), pp 467-488, 1982.

Feynman, R. Foundations of Physics, Vol. 16, No. 530, 1986.

Gell-Mann, M. What is Complexity? Complexity, Vol. 1:1, pp 16-19. New York: John Wiley and Sons, Inc, 1995.

Gödel, K. Über formal unenscheidbare Sätze der Principa Mathematica und verwandte Systeme, Monatsheftefür Mathematik und Physik, Vol. 38, pp 173-198. The first incompleteness theorem originally appeared as Theorem VI.

Hawking, S. The Universe in a Nutshell. New York: Bantam, 2001.

Hebb, D.O. The Organization of Behavior: A Neurophysiological Theory. New York: John Wiley and Sons, Inc, 1949.

Hemphill, J. and A. Dieters. DNA Computation in Mammalian Cells: MicroRNA Logic Operations, Journal of the American Chemical Society, July 17, Vol. 135, No. 28, pp 10512-8, 2013.

Holland, J.H. Hidden Order: How Adaptation Builds Complexity. New York: Perseus Books, 1995.

Ivars, P. Using quantum computation, in theory, to speed up database searches, Science News Online, August 31, 1996. Retrieved on July 10, 2013: http://www.sciencenews.org/sn_arch/8_31_96/bob2.htmQuantum-Quick Queries

Kleene, S.C. Mathematical Logic. New York: John Wiley, p. 250, 1967.

Kolmogorov, A.N. Three approaches to the quantitative definition of information, Problems of Information Transmission, Vol. 1, pp 1-11, 1965.

Kuhn, T. The Structure of Scientific Revolutions. Chicago: University of Chicago Press, 1962.

Kwiat, P.G and A.G. White. "Interaction-Free” Measurements: The In's and Out's of Quantum Interrogation, Physics Division, Progress Report, p 116-121, 1997-1998.

Leibniz, G.W. Discours, part 6; Principles of Nature and Grace, part 7; Monadology, part 33-35, 1714.

Leverington, D. A Basic Introduction to Feedforward Backpropagation Neural Networks, 2009. Retrieved on October 25, 2013, from http://www.webpages.ttu.edu/dleverin/neural_network/neural_networks.html.

Manin, Y.I. Vychisl i moeinevychislimoe [Computable and Noncomputable] (in Russian). Sov. Radio. pp 13-15, 1980.

McCulloch, W. S. and W. H. Pitts. A logical calculus of the ideas immanent in nervous activity, Bulletin of Mathematical Biophysics, Vol. 5, pp 115-133, 1943.

Megidish, E. et al. Entanglement Swapping between Photons that have Never Coexisted, Physical Review Letters, Vol. 110, p 21. Retrieved on July 18, 2013 from http://prl.aps.org/abstract/PRL/v110/i21/e210403

Nadin, M. Mind - Anticipation and Chaos (from the series Milestones in Thought and Discovery). Stuttgart: Belser Presse, 1986/1991.

Nadin, M. Anticipation - The End Is Where We Start From. Basel: Lars Muller, 2003a.

Nadin, M. Not everything we know we learned. In: M. Butz, (Ed.), Adaptive Behavior in Anticipatory Learning Systems LNAI 2684. Heidelberg: Bertelsman Springer, pp 23-43, $2003 \mathrm{~b}$.

Nadin, M. Anticipation and dynamics: Rosen's anticipation in the perspective of time, International Journal of General Systems (special issue). London: Taylor and Francis, Vol. 39, No. 1, pp 3-33, 2010a. 
Nadin, M. A Science of Change, in Vivires Cambiar, by Dr. F. Chorda, an interpretation of the thought of Mihai Nadin. Barcelona: Anthropos, pp 127-166, 2010b.

Nadin, M. Reassessing the Foundations of Semiotics: Preliminaries. In: A. Loula and J. Queiroz (Eds.), International Journal of Signs and Semiotic Systems, Vol. 2, no.1, pp 1-31, 2012.

Nadin, M. G-Complexity, Quantum Computation and Anticipatory Processes, Computer Communication \& Collaboration, Vol. 2, No. 1, pp 1-28, 2014. Toronto: BAP, Academic Research Council of Canada.

Neumann, J. von. The general and logical theory of automata, published (1951). In: L.A. Jeffress, (Ed.), Cerebral Mechanisms in Behavior - The Hixon Symposium. New York: John Wiley \& Sons, pp 1-31, 1948.

Poincare, H. Science and Hypothesis. London/New York: Walter Scott Publishing Co., 1904

Poplavskii, R.P. Thermodynamical models of information processing (in Russian), Uspekhi Fizicheskikh Nauk, Vol. 115, No. 3, pp 465-501, 1975.

Preskill, J. Physics 219/Computer Science 219, a course on quantum information and computation, 2011. See: http:/ /www.theory.caltech.edu/ preskill/ph219/ph219_2011

Prigogine, I. The End of Certainty. New York: Free Press, 1997.

Rawbone, P. (undated) Environmental Computation. A nonalgorithmic approach to AI. See: http://comm.cs.unb.ca/ sepics_wiki/uploaded_files/b/bf/Pete_Rawbone_Presentation1.pdf

Rosen, R. Anticipatory Systems. Oxford: Pergamon Press, 1985.

Rosen, R. Epistemology of Complexity. In: Kelso, Mandell, and Schlesinger (Eds.), Prologue in Dynamic Patterns of Complex Systems. Singapore: World Scientific, pp 7-30, 1988.

Schrödinger, E. What Is Life? Cambridge: Cambridge University Press, 1944.

Shomrat, T. and M. Levin. An automated training paradigm reveals long-term memory in planaria and its persistence through head regeneration, The Journal of Experimental Biology, 2013. Retrieved on July 7, 2013 from http:/ /jeb.biologists.org/content/early/2013/06/27/jeb.087809.abstract

Shor, P. Polynomial-Time Algorithms for Prime Factorization and Discrete Logarithms on a Quantum Computer, SIAM Journal of Computing, Vol. 26, no. 5, pp 1484-1509, 1994.

Waldrop, M.M. Complexity, New York: Simon \& Schuster, 1993.

Weyl, H. The Open World: Three Lectures on the Metaphysical Implications of Science. New Haven: Yale University Press, 1932.

Wheeler, J.A. Information, physics, quantum. The search for links. In: W. Zurek (Ed.), Complexity, Entropy and the Physics of Information. Redwood City CA: Addison Wesley, p. 5, 1990.

Wolfram, S. A New Kind of Science. Champaign IL: Wolfram Media, 2002.

Wolpert, D.H. Computational capabilities of physical systems, Physical Review E, Vol. 65, p 016128, 2001.

Zuse, K. Rechnender Raum. Schriftenzur Datenverarbeitung, Vol. 1. Braunschweig: Vieweg \& Sohn, 1969. 